\title{
List Circular Coloring of Trees and Cycles
}

\section{André Raspaud ${ }^{1}$ and Xuding $\mathrm{Zhu}^{2,3}$}

${ }^{1}$ LaBRI, UNIVERSITÉ BORDEAUXI 33405 TALENCE CEDEX, FRANCE

E-mail: raspaud@labri.fr

2 DEPARTMENT OF APPLIED MATHEMATICS NATIONAL SUN YAT-SEN UNIVERSITY KAOHSIUNG, TAIWAN 80424

E-mail: zhu@math.nsysu.edu.tw

${ }^{3}$ NATIONAL CENTER FOR THEORETICAL SCIENCES,

TAIWAN

Received February 7, 2006; Revised October 17, 2006

Published online 22 February 2007 in Wiley InterScience(www.interscience.wiley.com). DOI 10.1002/jgt.20234

Abstract: Suppose $G=(V, E)$ is a graph and $p \geq 2 q$ are positive integers. A $(p, q)$-coloring of $G$ is a mapping $\phi: V \rightarrow\{0,1, \ldots, p-1\}$ such that for any edge $x y$ of $G, q \leq|\phi(x)-\phi(y)| \leq p-q$. A color-list is a mapping $L: V \rightarrow \mathcal{P}(\{0,1, \ldots, p-1\})$ which assigns to each vertex $v$ a set $L(v)$ of permissible colors. An $L-(p, q)$-coloring of $G$ is a $(p, q)$-coloring $\phi$ of $G$ such that for each vertex $v, \phi(v) \in L(v)$. We say $G$ is $L-(p, q)$-colorable if there exists an $L-(p, q)$-coloring of $G$. A color-size-list is a mapping $\ell$ which assigns to each vertex $v$ a non-negative integer $\ell(v)$. We say $G$ is $\ell-(p, q)$-colorable if for every color-list $L$ with $|L(v)|=\ell(v), G$ is $L-(p, q)$-colorable. In this article, we consider list circular coloring of trees and cycles. For any tree $T$ and for any $p \geq 2 q$, we present a necessary and sufficient condition for $T$ to be

Contract grant sponsor: National Science Council; Contract grant number: NSC942115-M-110-001

Part of the work was done during the stay of X.Z. at LaBRI, supported by the French Ministry of Education.

Journal of Graph Theory

(c) 2007 Wiley Periodicals, Inc. 
$\ell$ - $(p, q)$-colorable. For each cycle $C$ and for each positive integer $k$, we present a condition on $\ell$ which is sufficient for $C$ to be $\ell-(2 k+1, k)$-colorable, and the condition is sharp. @ 2007 Wiley Periodicals, Inc. J Graph Theory 55: 249-265, 2007

Keywords: circular chromatic number, list circular coloring, circular list chromatic number

\section{INTRODUCTION}

Suppose $G=(V, E)$ is a graph and $p \geq 2 q$ are positive integers. A $(p, q)$-coloring of $G$ is a mapping $\phi: V \rightarrow\{0,1, \ldots, p-1\}$ such that for every edge $e=x y$, $q \leq|\phi(x)-\phi(y)| \leq p-q$. The circular chromatic number of $G$ is defined as $\chi_{c}(G)=\inf \{p / q$ : there exists a $(p, q)$-coloring of $G\}$. It is known [13] that for any finite graph $G$, the infimum in the definition is always attained and hence can be replaced by the minimum. Moreover, for any graph $G, \chi(G)-1<\chi_{c}(G) \leq \chi(G)$. So $\chi_{c}(G)$ is a refinement of $\chi(G)$, and it contains more information about the structure of $G$. The concept of the circular chromatic number of a graph was first introduced by Vince [11] under the name "star chromatic number," and has been studied extensively in the past decade. Readers are referred to $[13,14]$ for surveys on this subject.

In this article, we are interested in the list version of circular coloring. Suppose $G=(V, E)$ is a graph. A color-list of $G$ is a mapping $L$ which assigns to each vertex $v$ of $G$ a set of non-negative integers. Suppose $p \geq 2 q$ are positive integers and $p>\max \bigcup_{v \in V} L(v)$. Then an $L-(p, q)$-coloring of $G$ is a $(p, q)$-coloring $\phi$ of $G$ such that for each vertex $v, \phi(v) \in L(v)$. We say $G$ is $L-(p, q)$-colorable if such a coloring exists. In case $L(v)=\{0,1, \ldots, p-1\}$ for each vertex $v$, then an $L-(p, q)$-coloring is equivalent to a $(p, q)$-coloring. In the following, when we write $L-(p, q)$-coloring, it is implicitly implied that $p>\max \bigcup_{v \in V} L(v)$.

A color-size-list is a mapping $\ell$ which assigns to each vertex $v$ of $G$ a non-negative integer $\ell(v)$. Given a color-size-list $\ell$, we say $G$ is $\ell$ - $(p, q)$-colorable if for every color-list $L$ with $|L(v)|=\ell(v)$ and with $\max \bigcup_{v \in V} L(v)<p, G$ is $L$ - $(p, q)$-colorable.

A color-size-list $\ell$ is called $k$-uniform if $\ell(x)=k$ for some constant $k$. If for every $k$-uniform color-size-list $\ell, G$ is $\ell$ - $(p, 1)$-colorable for every $p$, then $G$ is called $k$-choosable. Suppose $t$ is a real number. If for every $k / q \geq t$, for every $p \geq k$ and for every $k$-uniform color-size-list $\ell, G$ is $\ell-(p, q)$-colorable, then we say $G$ is circular $t$-choosable. The list chromatic number $\chi_{l}(G)$ of $G$ is defined as

$$
\chi_{l}(G)=\inf \{t: G \text { is } t \text {-choosable }\} .
$$

The circular list chromatic number $\chi_{c, l}(G)$ of $G$ is defined as

$$
\chi_{c, l}(G)=\inf \{t: G \text { is circular } t \text {-choosable }\} .
$$

It is proved in [15] that for any graph $G, \chi_{c, l}(G)>\chi_{l}(G)-1$. On the other hand, $\chi_{c, l}(G)$ could be arbitrarily larger than $\chi_{l}(G)$. 
In this article, we consider color-size-lists $\ell$ that are not uniform. Given a graph $G$ and a pair of integers $p, q$, the question is for which color-size-lists $\ell, G$ is $\ell$ - $(p, q)$-colorable. We consider the case that $G$ is either a tree or a cycle. For each tree $T$, we present a condition on $\ell$ which is necessary and sufficient for $T$ to be $\ell-(p, q)$-colorable. For each cycle $C$, we present a condition on $\ell$ which is sufficient for $C$ to be $\ell-(2 k+1, k)$-colorable.

List coloring of a graph originally arose from the need in the inductive proofs of coloring results, and it is a useful tool in the proof of some coloring theorems about planar graphs, see $[9,10]$. Circular list coloring is also motivated by the need in the inductive proofs of circular coloring results. For example, the circular chromatic number of planar graphs of large odd girth is studied extensively in the literature $[1,2,4,7,12]$. A common feature of the proofs in these papers is that one needs to extend a $(2 k+1, k)$-coloring of a special subgraph $G^{\prime}$ to the entire graph $G$, where $G^{\prime}=G-T$, where $T$ is a tree. To extend a $(2 k+1, k)$-coloring $f$ of $G^{\prime}$ to $G$, it amounts to find an $L-(2 k+1, k)$-coloring of $T$, where $L$ is defined as follows: If $x$ is a leaf vertex of $T$, then $x$ is colored already, and hence $L(x)=\{f(x)\}$, if $x$ is not a leaf vertex, then let $L(x)=\{0,1, \ldots, 2 k\}$. The trees $T$ used in [2] and [7] are paths, and the trees $T$ used in $[1,4,12]$ are star-like trees. The results obtained so far concerning the circular chromatic number of planar graphs of large girth is still far from a conjectured value $[3,5,6]$. To further improve the above results, it seems that one inevitably needs to consider how to extend a coloring of $G-H$ to $G$, where $H$ is a more complicated subgraph. The result in this article is motivated by the study of this problem. We hope that the result in this article can be applied to obtain better results concerning this problem. On the other hand, like the study of list coloring problems, the study of circular list coloring is an interesting problem by itself.

\section{COLORING THE TREES}

First we introduce some notation that will be used throughout the article. Suppose $p$ is a positive integer. Then for any integer $t,[t]_{p}$ denotes the remainder of $t$ upon the division by $p$, that is, $[t]_{p}$ is the unique integer $0 \leq t^{\prime}<p$ such that $t-t^{\prime}$ is a multiple of $p$. In $(p, q)$-colorings of graphs, the color set is $Z_{p}=\{0,1, \ldots, p-1\}$. The summation in colors are all modulo $p$, and any integer $t$ for which $[t]_{p}=i$ can be used to represent the color $i$. For example, when we say "color a vertex $x$ with color $2 p$," it means to color $x$ with color 0 . Moreover, the colors are viewed to form a circle, that is, the integers $0,1, \ldots, p-1$ are cyclically ordered. If $a, b \in$ $\{0,1, \ldots, p-1\}$, then $[a, b]_{p}$ denotes the set of cyclically consecutive elements of the set $\{0,1, \ldots, p-1\}$ from $a$ to $b$. That is, $[a, b]_{p}=\left\{t:[t-a]_{p} \leq[b-a]_{p}\right\}$. For example, $[2,5]_{p}=\{2,3,4,5\}$ and $[5,2]_{p}=\{5,6, \ldots, p-1,0,1,2\}$. The set $[a, b]_{p}$ is called an interval of colors. For convenience, for arbitrary integers $a, b$ (not necessarily between 0 and $p$ ), let $[a, b]_{p}=\left[[a]_{p},[b]_{p}\right]_{p}$. The intervals $(a, b)_{p}$, $(a, b]_{p}$, and $[a, b)_{p}$ are defined similarly. The length $\ell\left([a, b]_{p}\right)$ of an interval $[a, b]_{p}$ is the number of integers in the interval and is equal to $[b-a]_{p}+1$. If the integer $p$ is 
clear from the context, then we may write $[a, b]$ for $[a, b]_{p}$. When considering $(p, q)$ colorings of graphs, we say two colors $i, j$ are adjacent if $q \leq|i-j| \leq p-q$. For two sets $A, B$ of colors, let $A+B=\left\{[a+b]_{p}: a \in A, b \in B\right\}$. Observe that when considering $(p, q)$-colorings of graphs, for a set $A$ of colors, $A+[q, p-q]_{p}$ is the set of colors which is adjacent to at least one color in $A$. The following CauchyDavenport Theorem is a well-known result in number theory.

Theorem 2.1. If $p$ is a prime number, then for any subsets $A, B$ of $Z_{p},|A+B| \geq$ $\min \{|A|+|B|-1, p\}$.

For our purpose, $p$ is usually not a prime number. However, most of the time we work with special sets $A, B$ of colors. For those special sets that we are interested in, the corresponding conclusion holds for non-prime $p$.

Lemma 2.2. Suppose $B$ is an interval of colors. For any set $A$ of colors, $|A+B| \geq$ $\min \{|A|+|B|-1, p\}$.

Proof. Suppose $A=\left[a_{1}, b_{1}\right] \cup\left[a_{2}, b_{2}\right] \cup \cdots \cup\left[a_{t}, b_{t}\right]$ and $B=[c, d]$. The intervals $\left(b_{1}, a_{2}\right),\left(b_{2}, a_{3}\right), \ldots,\left(b_{t}, a_{1}\right)$ are the "gaps" of $A$. It is known (see [8]) that

$$
\begin{aligned}
& A+B=\{0,1, \ldots, p-1\}, \text { or } \\
& A+B=\left[a_{1}+c, b_{1}+d\right] \cup\left[a_{2}+c, b_{2}+d\right] \cup \cdots \cup\left[a_{t}+c, b_{t}+d\right] .
\end{aligned}
$$

If there is a gap, say $\left(b_{1}, a_{2}\right)$, of size at least $|B|$, then

$$
\left[a_{1}+c, b_{1}+d\right],\left[a_{2}+c, b_{2}+c\right], \ldots,\left[a_{t}+c, b_{t}+c\right]
$$

are pair-wise disjoint subsets of $A+B$. Therefore

$$
\begin{aligned}
|A+B| & \geq\left|\left[a_{1}+c, b_{1}+d\right]\right|+\left|\left[a_{2}+c, b_{2}+c\right]\right|+\cdots+\left|\left[a_{t}+c, b_{t}+c\right]\right| \\
& =|A|+|B|-1 .
\end{aligned}
$$

If each of the gaps of $A$ has size less than $B$, then it is easy to see that $A+B=$ $\{0,1, \ldots, p-1\}$, and hence $|A+B|=p$.

In this section, we prove the following theorem which characterizes the colorsize-lists $\ell$ of a tree $T$ for which $T$ is $\ell-(p, q)$-colorable.

Theorem 2.3. Suppose $T$ is a tree, $p \geq 2 q$ are positive integers and $\ell: V(T) \rightarrow$ $\{0,1,2, \ldots, p\}$ is a color-size-list. Then $T$ is $\ell-(p, q)$-colorable if and only if for each subtree $T^{\prime}$ of $T$,

$$
\sum_{v \in T^{\prime}} \ell(v) \geq 2\left(\left|V\left(T^{\prime}\right)\right|-1\right) q+1
$$

The "only if" part of Theorem 2.3 follows from the following lemma. Journal of Graph Theory DOI 10.1002/jgt 
Lemma 2.4. Suppose $\ell$ is a color-size-list of a tree $T=(V, E)$. If $\sum_{x \in T} \ell(x)<$ $2(|V|-1) q+1$, then there is a color-list $L$ such that $L(x)$ is an interval of colors with $|L(x)|=\ell(x)$ for each vertex $x$, and $T$ is not $L-(p, q)$-colorable.

Proof. We prove Lemma 2.4 by induction on $|V|$. If $V=\{v\}$, then the condition says that $\ell(v)=0$, and hence $L(v)=\emptyset$ for the only vertex $v$ of $T$. Then of course, $T$ is not $L-(p, q)$-colorable.

Assume $|V| \geq 2$. Let $v$ be a leaf of $T$. Let $u$ be the neighbor of $v$. If $\ell(u)+\ell(v) \leq$ $2 q$, then let $L(v)=[0, \ell(v)-1]_{p}$ and let $L(u)=[\ell(v)+p-q, \ell(v)+p-q+$ $\ell(u)-1]_{p}$, and for $x \neq u, v$, let $L(x)$ be any interval of colors for which $|L(x)|=$ $\ell(x)$. Observe that no color in $L(u)$ is adjacent to a color in $L(v)$. So $T$ is not $L$ - $(p, q)$-colorable.

Assume $\ell(u)+\ell(v) \geq 2 q+1$. If $\ell(v) \geq 2 q$, then let $\ell^{\prime}$ be the color-size-list of $T-v$, defined as $\ell^{\prime}(x)=\ell(x)$ for all $x$. If $\ell(v) \leq 2 q-1$, then let $\ell^{\prime}$ be the colorsize-list of $T-v$, defined as $\ell^{\prime}(x)=\ell(x)$ if $x \neq u$, and $\ell^{\prime}(u)=\ell(u)+\ell(v)-2 q$. In any case, $\sum_{x \in T-v} \ell^{\prime}(v) \leq \sum_{x \in T} \ell(x)-2 q$. Therefore $\ell^{\prime}$ satisfies the condition of Lemma 2.4. By induction hypothesis, there is a color-list $L^{\prime}$ such that $L^{\prime}(x)$ is an interval of size $\ell^{\prime}(x)$ for each vertex $x$, and $T-v$ is not $L^{\prime}-(p, q)$-colorable. Assume $L^{\prime}(u)=[c, d]$.

If $\ell(v) \geq 2 q$, then let $L$ be any extension of $L^{\prime}$. Any $L-(p, q)$-coloring induces an $L^{\prime}-(p, q)$-coloring of $T-v$. Therefore, $T$ is not $L-(p, q)$-colorable. If $\ell(v) \leq 2 q-$ 1 , then let $L(v)=[c-q, c+\ell(v)-q-1], L(u)=[d-\ell(u)+1, d]$ and $L(x)=$ $L^{\prime}(x)$ for $x \neq u, v$. Observe that $L(v)+[q, p-q]=[c, c+\ell(v)+p-2 q-1]$. Since $|[c, d]|=\ell(u)+\ell(v)-2 q$, we conclude that

$$
(L(v)+[q, p-q]) \cap L(u)=[c, d] .
$$

Therefore if $\phi$ is an $L$ - $(p, q)$-coloring of $T$ such that $\phi(x) \in L(x)$ for all $x$, then $\phi(u) \in[c, d]$, that is, the restriction of $\phi$ to $T-v$ is an $L^{\prime}-(p, q)$-coloring of $T-v$, contrary to the assumption that $T-v$ is not $L^{\prime}-(p, q)$-colorable. Therefore $T$ is not $L$ - $(p, q)$-colorable.

The "if" part of Theorem 2.3 follows from the following lemma.

Lemma 2.5. Assume $L$ is a color-list of $T$. If for each subtree $T^{\prime}$ of $T$,

$$
\sum_{v \in T^{\prime}}|L(v)| \geq 2\left(\left|V\left(T^{\prime}\right)\right|-1\right) q+1
$$

then $T$ is $L-(p, q)$-colorable.

Proof. We prove Lemma 2.5 by induction on $|V(T)|$. Assume $L$ is a colorlist of $T$ such that for each subtree $T^{\prime}$ of $T, \sum_{v \in T^{\prime}}|L(v)| \geq 2\left(\left|V^{\prime}\right|-1\right) q+1$. If $|V(T)|=1$, then the condition implies that $L(v) \neq \emptyset$ for the only vertex $v$ of $T$. Hence $T$ is $L-(p, q)$-colorable. Assume $|V(T)| \geq 2$. Let $v$ be a leaf of $T$. Let $u$ be the neighbor of $v$. Consider the edge $e=u v$, which is a subtree of $T$. The condition of Lemma 2.5 implies that $|L(u)|+|L(v)| \geq 2 q+1$. 
Similarly as before, $L(v)+[q, p-q]_{p}$ is the set of colors each of which is adjacent to at least one color of $L(v)$. By Lemma 2.2, $\left|L(v)+[q, p-q]_{p}\right| \geq$ $\min \{|L(v)|+p-2 q, p\}$. If $\left|L(v)+[q, p-q]_{p}\right|=p$, then let $L^{\prime}$ be the restriction of $L$ to $T-v$. Any $L^{\prime}-(p, q)$-coloring $\phi$ of $T-v$ can be extended to an $L$ $(p, q)$-coloring of $T$. Otherwise $\left|L(v)+[q, p-q]_{p}\right| \geq|L(v)|+p-2 q$. Let $L^{\prime}$ be the color-list of $T-v$ defined as $L^{\prime}(x)=L(x)$ for $x \neq u$ and $L^{\prime}(u)=L(u) \cap$ $\left(L(v)+[q, p-q]_{p}\right)$. Then $\left|L^{\prime}(u)\right| \geq|L(u)|+|L(v)|-2 q$. Straightforward calculation shows that $L^{\prime}$ satisfies the condition of Lemma 2.5. Therefore $T-v$ has an $L^{\prime}-(p, q)$-coloring $\phi$. As $\phi(u) \in L^{\prime}(u) \subseteq L(v)+[q, p-q]_{p}$, so $\phi(u)$ is adjacent to some color in $L(v)$. Hence $\phi$ can be extended to an $L-(p, q)$-coloring of $T$.

Theorem 2.6. Given a tree $T$, positive integers $p \geq 2 q$, and a color-size-list $\ell$ for $T$, it can be determined in linear time whether or not $T$ is $\ell$ - $(p, q)$-colorable.

Proof. Let $v$ be a leaf vertex of $T$ and let $u$ be the unique neighbor of $v$. If $\ell(u)+$ $\ell(v) \leq 2 q$, then $T$ is not $\ell-(p, q)$-colorable, by Theorem 2.3. Assume $\ell(u)+\ell(v) \geq$ $2 q+1$. Delete $v$, and let $\ell^{\prime}(u)=\ell(u)+\ell(v)-2 q$ and $\ell^{\prime}(x)=\ell(x)$ for $x \neq u, v$. It follows from Theorem 2.3 that $T$ is $\ell-(p, q)$-colorable if and only if $T-v$ is $\ell^{\prime}-(p, q)$-colorable. By repeatedly deleting leaf vertices of $T$, one determines in linear time whether or not $T$ is $\ell-(p, q)$-colorable.

\section{COLORING THE CYCLES}

In the remainder of this article, we consider list coloring of cycles. Given a cycle $X=\left(x_{0}, x_{1}, \ldots, x_{n-1}\right)$, the vertices are also considered as cyclically ordered. The additions on the indices of the vertices of the cycle are modulo $n$. The intervals $[i, j]_{n},(i, j)_{n},[i, j)_{n},(i, j]_{n}$ are defined in the same way as the intervals of colors.

The following result is the main theorem of this section.

Theorem 3.1. Let $k \geq 1$ be an integer, and $X=\left(x_{0}, x_{1}, \ldots, x_{n-1}\right)$ be a cycle of length $n \geq 2 k+1$. Suppose $\ell: V(X) \rightarrow\{0,1,2, \ldots, 2 k+1\}$ is a color-size-list for $X$. Then $X$ is $\ell-(2 k+1, k)$-colorable if the following conditions hold:

1. For each interval $\left[j, j^{\prime}\right]_{n}$ of length $m, \sum_{t \in\left[j, j^{\prime}\right]_{n}} \ell\left(x_{t}\right) \geq 2(m-1) k+1$.

2. $\sum_{t=0}^{n-1} \ell\left(x_{t}\right) \geq 2 n k+1$.

Moreover, Condition (1) is necessary for $X$ to be $\ell-(2 k+1, k)$-colorable, and in case $X$ is an odd cycle, Condition (2) is sharp.

The necessity of Condition 1 follows from Lemma 2.4, because if $X$ is $\ell-(2 k+$ $1, k)$-colorable, then each subtree (which is a path) must be $\ell-(2 k+1, k)$-colorable.

If $X=\left(x_{0}, x_{1}, \ldots, x_{n-1}\right)$ is an odd cycle, then Condition (2) is sharp in the following sense: There is a color-size-list $\ell$ which satisfies Condition (1) and $\sum_{t=0}^{n-1} \ell\left(x_{t}\right)=2 n k$, however, $X$ is not $\ell-(2 k+1, k)$-colorable. For example, if $L\left(x_{i}\right)=[1,2 k]$ for each $i$, then $\ell\left(x_{i}\right)=\left|L\left(x_{i}\right)\right|$ satisfies Condition (1), and 
$\sum_{t=0}^{n-1} \ell\left(x_{t}\right)=2 n k$. However, $X$ is not $L-(2 k+1, k)$-colorable, because an $L-(2 k+$ $1, k)$-coloring $\phi$ of $X$ is equivalent to a homomorphism from $X$ to $C_{2 k+1}-\{0\}$, and $C_{2 k+1}-\{0\}$ is a bipartite graph. However, Condition (2) is not a necessary condition. There are color-size-lists $\ell$ which violates Condition (2) and yet $X$ is $\ell$ - $(2 k+1, k)$-colorable. For example, suppose $X=\left(x_{0}, x_{1}, x_{2}, x_{3}, x_{4}\right)$ is a 5-cycle. Let $\ell\left(x_{0}\right)=3, \ell\left(x_{1}\right)=5$, and $\ell\left(x_{i}\right)=4$ for $i \geq 2$. Then $X$ is $\ell-(5,2)$-colorable, although Condition (2) is violated.

Given a color-list $L$ for $X$, let $F(i)=\{0,1, \ldots, 2 k\}-L\left(x_{i}\right)$. So $F(i)$ is the set of forbidden colors for $x_{i}$. It seems to be more convenient for us to work with the forbidden colors for each vertex. Thus we restate the main result in terms of forbidden colors. Formally, we define a forbidden color assignment, abbreviated as FCA, as a pair $(X, F)$ such that $X=\left(x_{0}, x_{1}, \ldots, x_{n-1}\right)$ is a cycle and $F$ is a mapping which assigns to each index $i$ a set $F(i)$ of forbidden colors for $x_{i}$. A FCA is valid if

1. For any interval $\left[j, j^{\prime}\right]_{n}$ of length $m, \sum_{s \in\left[j, j^{\prime}\right]_{n}}|F(s)| \leq 2 k+m-1$.

2. $\sum_{i=0}^{n-1}|F(i)| \leq n-1$.

Given a FCA $(X, F)$, we say a $(2 k+1, k)$-coloring $\phi$ of $X$ is $\operatorname{good}$ for $(X, F)$ if for any $i, \phi\left(x_{i}\right) \notin F(i)$. Our main result can be stated as follows:

Theorem 3.2. If $(X, F)$ is a valid $F C A$, then there is a good $(2 k+1, k)$-coloring for $(X, F)$.

In the remainder of this article, we shall be only considering $(2 k+1, k)$-colorings of graphs. For simplicity, we refer a $(2 k+1, k)$-coloring simply as a coloring.

Given a FCA $(X, F)$, let

$$
\Gamma_{F}=\{(i, j): 0 \leq i \leq n-1,0 \leq j \leq 2 k, j \in F(i)\} .
$$

Given a coloring $\phi$ of $X$, let

$$
\Gamma_{\phi}=\left\{(i, j): 0 \leq i \leq n-1,0 \leq j \leq 2 k, j=\phi\left(x_{i}\right)\right\} .
$$

To prove Theorem 3.2, we need to find a coloring $\phi$ such that $\Gamma_{\phi} \cap \Gamma_{F}=\emptyset$.

It is helpful to have a picture for the understanding of the proof below: We construct a graph $G$ whose vertex set is partitioned into $n$ columns $B_{i}=\{(i, j)$ : $0 \leq j \leq 2 k\}$, for $i=0,1, \ldots, n-1$. Each vertex $(i, j)$ in $B_{i}$ is connected to two vertices in $B_{i+1}$, namely $(i+1, j+k)$ and $(i+1, j+k+1)$, where the summation in the first coordinate is modulo $n$, and the summation in the second coordinate is modulo $2 k+1$. A coloring $\phi$ corresponds to a cycle of $G$ which intersects each column $B_{i}$ exactly once. We call such a cycle of $G$ a coloring cycle. The set $\Gamma_{F}$ is the set of forbidden vertices in $G$. We need to find a "coloring cycle" which avoids the forbidden vertices $\Gamma_{F}$. Figure 1 below is an example of the graph $G$ with $k=3$ and $n=11$. There are edges between vertices in $B_{10}$ and $B_{0}$, however, for simplicity, these edges are not shown in the figure. The thick edge indicates a coloring cycle. 


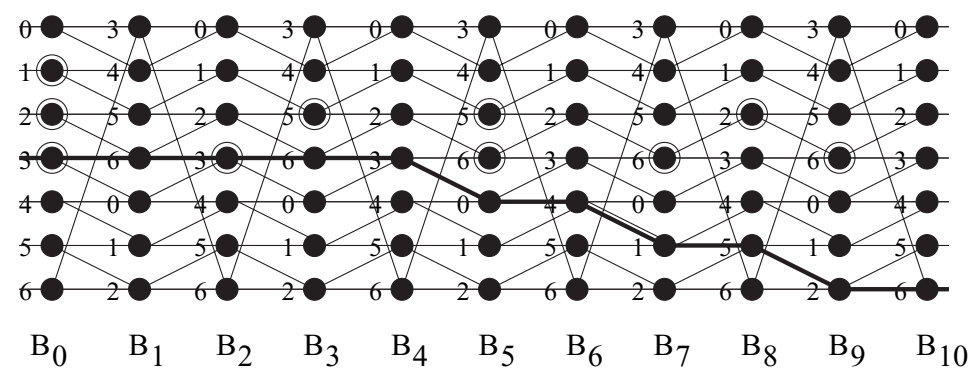

Figure 1. An example graph $G$.

(The two ends should meet, i.e., the vertex 6 in Column $B_{10}$ is adjacent to the vertex 3 in Column $B_{0}$.) Circled vertices indicate vertices in $F$, that is,

$$
\begin{aligned}
& F_{0}=\{1,2,3\}, F_{2}=\{3\}, F_{3}=\{5\}, F_{5}=\{5,6\}, F_{7}=\{6\}, \\
& F_{8}=\{2\}, F_{9}=\{6\}, F_{1}=F_{4}=F_{6}=F_{10}=\emptyset
\end{aligned}
$$

Observe that the coloring cycle indicated by the thick edges in Figure 1 intersects the "forbidden vertices." So this coloring is not a good coloring.

We need to define some notation so that we can talk about the "shape" of the set of forbidden vertices.

Suppose $(X, F)$ is a valid FCA, where $X=\left(x_{0}, x_{1}, \ldots, x_{n-1}\right)$. We say a column $B_{i}$ is infected if $B_{i}$ contains at least one forbidden vertex, that is, $F(i) \neq \varnothing$. We say a column $B_{i}$ is seriously infected if $B_{i}$ contains at least two forbidden vertices, that is, $|F(i)| \geq 2$.

Let $J_{F}$ be the set of indices of the infected columns, and let $I_{F}$ be the set of indices of the seriously infected columns, that is,

$$
\begin{aligned}
& J_{F}=\{0 \leq i \leq n-1: F(i) \neq \emptyset\}, \\
& I_{F}=\{i:|F(i)| \geq 2\} .
\end{aligned}
$$

Let $|F|=\sum_{i=0}^{2 k}|F(i)|$.

For $i \in J_{F}$, let $q_{i}^{F}$ be the smallest positive integer such that $i+q_{i}^{F} \in J_{F}$. For $i \in I_{F}$, let $p_{i}^{F}$ be the smallest positive integer such that $i+p_{i}^{F} \in I_{F}$.

We shall prove that for a counterexample, $I_{F} \neq \varnothing$ (Lemma 4.4). Assume $i \in I_{F}$ and

$$
\left[i, i+p_{i}^{F}\right] \cap J_{F}=\left\{\beta_{0}^{i}, \beta_{1}^{i}, \ldots, \beta_{t_{i}}^{i}\right\}
$$

where $\beta_{0}^{i}=i, \beta_{t_{i}}^{i}=i+p_{i}^{F}$ and for $1 \leq j \leq t_{i}-1, \beta_{j}^{i} \in\left(\beta_{j-1}^{i}, \beta_{j+1}^{i}\right)_{n}$. Let

$$
s_{i}^{F}=\sum_{j=0}^{t_{i}-1} j \times \ell\left(\left[\beta_{j}^{i}, \beta_{j+1}^{i}\right)_{n}\right) .
$$


For the example in Figure 1, the corresponding parameters for this FCA are

$$
\begin{aligned}
& J_{F}=\{0,2,3,5,7,8,9\}, I_{F}=\{0,5\}, \\
& q_{0}^{F}=2, q_{2}^{F}=q_{3}^{F}=1, q_{5}^{F}=2, q_{7}^{F}=q_{8}^{F}=1, q_{9}^{F}=2, p_{0}^{F}=5, p_{5}^{F}=6, \\
& \beta_{0}^{0}=0, \beta_{1}^{0}=2, \beta_{2}^{0}=3, \beta_{3}^{0}=5 ; \beta_{0}^{5}=5, \beta_{1}^{5}=7, \beta_{2}^{5}=8, \beta_{3}^{5}=9, \beta_{4}^{5}=0, \\
& s_{0}^{F}=0 \times 2+1 \times 1+2 \times 2=5, s_{5}^{F}=0 \times 2+1 \times 1+2 \times 1+3 \times 2=9 .
\end{aligned}
$$

A sketch of the proof of Theorem 3.2. Assume Theorem 3.2 is not true, and assume that $(X, F)$ is a counterexample to Theorem 3.2 such that among all the counterexamples,

(a) : $|X|=n$ is minimum.

(b) : Subject to (a), $|F|$ is minimum.

(c) : Subject to (a) and (b), $\sum_{i \in I_{F}}|F(i)|$ is minimum.

(d) : Subject to (a), (b), and (c), $\sum_{i \in I_{F}} s_{i}^{F}$ is minimum.

We call such a counterexample a minimum counterexample. The key step in proving Theorem 3.2 is to show that if $(X, F)$ is a minimum counterexample, then $|F(i)| \geq q_{i}^{F}$ for all $i \in J_{F}$. This implies that

$$
n=\sum_{i \in J_{F}} q_{i}^{F} \leq \sum_{j=0}^{n-1}|F(j)|,
$$

which is in contrary to our assumption that $F$ is a valid FCA.

Definition 3.3. Suppose $(X, F)$ and $\left(X^{\prime}, F^{\prime}\right)$ are two $F C A$ 's. If the existence of a good coloring for $\left(X^{\prime}, F^{\prime}\right)$ implies the existence of a good coloring for $(X, F)$, then we say $\left(X^{\prime}, F^{\prime}\right)$ dominates $(X, F)$.

We shall prove that if for some $i \in J_{F},|F(i)|<q_{i}^{F}$, then there is a valid FCA $\left(X^{\prime}, F^{\prime}\right)$ dominating $(X, F)$ such that one of the following is true:

- $\left|X^{\prime}\right|<|X|$.

- $\left|X^{\prime}\right|=|X|$ and $\left|F^{\prime}\right|<|F|$.

- $\left|X^{\prime}\right|=|X|,\left|F^{\prime}\right|=|F|$ and $\sum_{i \in I_{F}}\left|F^{\prime}(i)\right|<\sum_{i \in I_{F}}|F(i)|$.

- $\left|X^{\prime}\right|=|X|,\left|F^{\prime}\right|=|F|$ and $\sum_{i \in I_{F}}\left|F^{\prime}(i)\right|=\sum_{i \in I_{F}}|F(i)|$ and $\sum_{i \in I_{F^{\prime}}} s_{i}^{F^{\prime}}<$ $\sum_{i \in I_{F}} s_{i}^{F}$.

This will be in contrary to the assumption that $(X, F)$ is a minimum counterexample. 


\section{SOME SPECIAL CASES OF THEOREM 3.2}

This Section proves Theorem 3.2 for $n=2 k+1, n=2 k+2$ and for the case that $I_{F}=\emptyset$.

Lemma 4.1. If $n=2 k+1$, then the conclusion of Theorem 3.2 holds.

Proof. Let $\phi$ be any coloring of $X$ (which obviously exists). For $i=$ $0,1, \ldots, 2 k$, let $\phi_{i}$ be the coloring of $X$ defined as $\phi_{i}(x)=\phi(x)+i$. Then $\Gamma_{\phi_{i}} \cap \Gamma_{\phi_{j}}=\emptyset$ if $i \neq j$. As $\left|\Gamma_{F}\right| \leq 2 k$, at least one of the $2 k+1$ colorings $\phi_{i}$ satisfies $\Gamma_{\phi_{i}} \cap \Gamma_{F}=\emptyset$.

Lemma 4.2. If $n=2 k+2$, then the conclusion of Theorem 3.2 holds.

Proof. Without loss of generality, we assume that $0 \notin F(0)$ and $1 \in F(0)$. Let $\phi$ be the coloring defined as $\phi\left(x_{i}\right)=1$ if $i$ is even and $\phi\left(x_{i}\right)=k+1$ if $i$ is odd. If $\Gamma_{F} \cap \Gamma_{\phi}=\{(0,1)\}$, then let $\phi^{\prime}\left(x_{i}\right)=\phi\left(x_{i}\right)$ for $i \neq 0$ and $\phi^{\prime}\left(x_{0}\right)=0$. As $0 \notin F(0)$, $\phi^{\prime}$ is a good coloring.

Assume $\left|\Gamma_{F} \cap \Gamma_{\phi}\right| \geq 2$. For $i=0,1, \ldots, 2 k$, let $\phi_{i}$ be the coloring of $X$ defined as $\phi_{i}(x)=\phi(x)+i$.

Since $\left|\Gamma_{F} \cap \Gamma_{\phi}\right| \geq 2$ and $\left|\Gamma_{F}\right| \leq 2 k+1$, there is an index $i$ such that $\Gamma_{\phi_{i}} \cap \Gamma_{F}=\emptyset$.

Lemma 4.3. Suppose for some index $i, F(i)=\{u\}$ and $F(i+1)=\{v\}$. Assume that $u$ and $v$ are not adjacent. Let $X^{\prime}=\left\{x_{0}^{\prime}, x_{1}^{\prime}, \ldots, x_{n-3}^{\prime}\right\}$ be a cycle of length $n-2$, and let $F^{\prime}$ be a FCA for $X^{\prime}$ defined as $F^{\prime}(j)=F(\tau(j))$, where $\tau:\{0,1, \ldots, n-$ $3\} \rightarrow\{0,1, \ldots, n-1\}$ is defined as

$$
\tau(j)= \begin{cases}j, & \text { if } j \leq i-1 \\ j+2, & \text { if } j \geq i\end{cases}
$$

Then $\left(X^{\prime}, F^{\prime}\right)$ is a valid $F C A$ and dominates $(X, F)$.

Proof. First we show that $\left(X^{\prime}, F^{\prime}\right)$ is valid. Consider an interval $\left[j, j^{\prime}\right]_{n-2}$ of length $m$. If $i \notin\left[j, j^{\prime}\right]_{n-2}$ or $i-1 \notin\left[j, j^{\prime}\right]_{n-2}$, then $\left[\tau(j), \tau\left(j^{\prime}\right)\right]_{n}$ also has length $m$, and

$$
\sum_{s \in\left[j, j^{\prime}\right]_{n-2}}\left|F^{\prime}(s)\right|=\sum_{s \in\left[\tau(j), \tau\left(j^{\prime}\right)\right]_{n}}|F(s)| \leq 2 k+m-1 .
$$

If $i-1, i \in\left[j, j^{\prime}\right]_{n-2}$, then $\left[\tau(j), \tau\left(j^{\prime}\right)\right]_{n}$ has length $m+2$, and

$$
\sum_{s \in\left[j, j^{\prime}\right]_{n-2}}\left|F^{\prime}(s)\right|=\sum_{s \in\left[\tau(j), \tau\left(j^{\prime}\right)\right]_{n}}|F(s)|-2 \leq 2 k+m+2-1-2=2 k+m-1 .
$$

Moreover, $\quad \sum_{s=0}^{n-3}\left|F^{\prime}(s)\right|=\sum_{s=0}^{n-1}|F(s)|-2 \leq n-1-2=n-3$. Therefore $\left(X^{\prime}, F^{\prime}\right)$ is valid.

Next we show that $\left(X^{\prime}, F^{\prime}\right)$ dominates $(X, F)$. Let $\phi^{\prime}$ be a good coloring for $\left(X^{\prime}, F^{\prime}\right)$. Recall that $F(i)=\{u\}$ and $F(i+1)=\{v\}$. As $u$ is not adjacent to $v$, and Journal of Graph Theory DOI 10.1002/jgt 
$\phi^{\prime}\left(x_{i-1}^{\prime}\right)$ is adjacent to $\phi^{\prime}\left(x_{i}^{\prime}\right)$, we conclude that either $\phi^{\prime}\left(x_{i-1}^{\prime}\right) \neq v$ or $\phi^{\prime}\left(x_{i}^{\prime}\right) \neq u$. If $\phi^{\prime}\left(x_{i-1}^{\prime}\right) \neq v$, then let $t \in\left\{\phi^{\prime}\left(x_{i-1}^{\prime}\right)+k, \phi^{\prime}\left(x_{i-1}^{\prime}\right)+k+1\right\} \backslash\{u\}$, and let

$$
\phi\left(x_{j}\right)= \begin{cases}\phi^{\prime}\left(x_{j}^{\prime}\right), & \text { if } j \leq i-1, \\ t, & \text { if } j=i, \\ \phi^{\prime}\left(x_{j-2}^{\prime}\right), & \text { if } j \geq i+1 .\end{cases}
$$

Then $\phi$ is a good coloring for $F$. If $\phi^{\prime}\left(x_{i}\right) \neq u$, then let $t \in\left\{\phi^{\prime}\left(x_{i}^{\prime}\right)+k, \phi^{\prime}\left(x_{i}^{\prime}\right)+k+\right.$ $1\} \backslash\{v\}$, and let

$$
\phi\left(x_{j}\right)= \begin{cases}\phi^{\prime}\left(x_{j}^{\prime}\right), & \text { if } j \leq i, \\ t, & \text { if } j=i+1, \\ \phi^{\prime}\left(x_{j-2}^{\prime}\right), & \text { if } j \geq i+2 .\end{cases}
$$

Then $\phi$ is a good coloring for $F$.

Lemma 4.4. Suppose $(X, F)$ is a FCA. If $I_{F}=\emptyset$, then there is a good coloring for $(X, F)$.

Proof. The proof is by induction on $|X|$. If $|X|=3$, then it is trivial. Assume $|X|=n \geq 4$ and the lemma is true for smaller cycles $X^{\prime}$. Since $\sum_{i=0}^{n-1}|F(i)| \leq$ $n-1$, we may assume (by adding more forbidden colors if necessary) that $|F(i)|=1$ for $i=0,1, \ldots, n-2$ and $F(n-1)=\emptyset$. If there is an index $i$ such that $F(i)=\{u\}, F(i+1)=\{v\}$ and $u$ is not adjacent to $v$, then the conclusion follows from Lemma 4.3 and the induction hypothesis. Assume for $i=0,1, \ldots, n-3$, the unique color in $F(i)$ is adjacent to the unique color in $F(i+1)$.

Let $\phi\left(x_{i}\right)$ be the unique color in $F(i)$, for $i=0,1, \ldots, n-2 k$. Let

$$
P=\left(\left(0, \phi\left(x_{0}\right)\right),\left(1, \phi\left(x_{1}\right)\right), \ldots,\left(n-2 k, \phi\left(x_{n-2 k}\right)\right) .\right.
$$

Then $P$ is a path in $G$ connecting $\left(0, \phi\left(x_{0}\right)\right)$ and $\left(n-2 k, \phi\left(x_{n-2 k}\right)\right)$. This path can be extended to a coloring cycle in $G$. To see this, we say a vertex $(j, s)$ in $B_{j}$ is reachable from $\left(n-2 k, \phi\left(x_{n-2 k}\right)\right)$ if there is a path $P^{\prime}$ in $G$ connecting $\left(n-2 k, \phi\left(x_{n-2 k}\right)\right)$ and $(j, s)$ such that $\left|P^{\prime} \cap B_{t}\right|=1$ for $t \in[n-2 k, j]_{n}$. Then $B_{n-2 k+1}$ contains two vertices that are reachable from $\left(n-2 k, \phi\left(x_{n-2 k}\right)\right)$, namely $\left(n-2 k+1, \phi\left(x_{n-2 k}\right)+k\right)$ and $\left(n-2 k+1, \phi\left(x_{n-2 k}\right)+k+1\right)$. Inductively, it is easy to show that for $s=1,2, \ldots, 2 k, B_{n-2 k+s}$ contains $s+1$ vertices that are reachable from $\left(n-2 k, \phi\left(x_{n-2 k}\right)\right)$. In particular, every vertex of $B_{0}$ is reachable from $\left(n-2 k, \phi\left(x_{n-2 k}\right)\right)$. Let $P^{\prime}$ be a path in $G$ connecting $\left(n-2 k, \phi\left(x_{n-2 k}\right)\right)$ and $\left(0, \phi\left(x_{0}\right)\right)$ such that $\left|P^{\prime} \cap B_{t}\right|=1$ for $t \in[n-2 k, 0]_{n}$. Then $P \cup P^{\prime}$ is a coloring cycle in $G$. Let $\phi$ be the coloring of $X$ corresponding to this coloring cycle. Then $\left|\Gamma_{\phi} \cap \Gamma_{F}\right| \geq n-2 k+1$.

For $i=0,1, \ldots, 2 k$, let $\phi_{i}$ be the coloring of $X$ defined as $\phi_{i}(x)=\phi(x)+i$. Since $\left|\Gamma_{F}\right| \leq n-1$, there is an index $i$ such that $\Gamma_{\phi_{i}} \cap \Gamma_{F}=\emptyset$.

A maximal color interval of $F(i)$ is an interval $\left[j, j^{\prime}\right]_{2 k+1} \subset F(i)$ such that $j-1 \notin$ $F(i)$ and $j^{\prime}+1 \notin F(i)$. 
Lemma 4.5. Suppose $(X, F)$ is a counterexample to Theorem 3.2 with $|X|$ minimum. Then for each $i, F(i)$ is either empty or consists of one maximal color interval.

Proof. Assume to the contrary that $F(n-2)$ consists of maximal color intervals $I_{1}=\left[a_{1}, b_{1}\right]_{2 k+1}, \ldots, I_{s}=\left[a_{s}, b_{s}\right]_{2 k+1}$, where $s \geq 2$. For $i=1,2, \ldots, s$, if $a_{i}=$ $b_{i}$, then let $J_{i}=\emptyset$. Otherwise, let $J_{i}=\left[a_{i}-k, b_{i}-k-1\right]_{2 k+1}$. Note that $\left|J_{i}\right|=$ $\left|I_{i}\right|-1$.

Let $X^{\prime}=\left(x_{0}^{\prime}, x_{1}^{\prime}, \ldots, x_{n-3}^{\prime}\right)$ be a cycle of length $n-2$. Define $F^{\prime}$ as follows: $F^{\prime}(i)=F(i)$ for $i=0,1, \ldots, n-4, F^{\prime}(n-3)=F(n-3) \cup J_{1} \cup \cdots \cup$ $J_{s} \cup F(n-1)$.

Similarly as the proof of Lemma 4.3 , one can prove that $\left(X^{\prime}, F^{\prime}\right)$ is a valid FCA. Now we derive a contradiction by showing that $\left(X^{\prime}, F^{\prime}\right)$ dominates $(X, F)$. Let $\phi^{\prime}$ be a good coloring for $\left(X^{\prime}, F^{\prime}\right)$. As $\phi^{\prime}\left(x_{n-3}^{\prime}\right) \notin F^{\prime}(n-3)$, it follows from the definition of $F^{\prime}(n-3)$ that there is a color $t \notin F(n-2)$ which is adjacent to $\phi^{\prime}\left(x_{n-3}^{\prime}\right)$. Let $\phi$ be defined by

$$
\phi\left(x_{i}\right)= \begin{cases}\phi^{\prime}\left(x_{i}^{\prime}\right), & \text { if } 0 \leq i \leq n-3 \\ \phi^{\prime}\left(x_{n-3}^{\prime}\right), & \text { if } i=n-1 \\ t, & \text { if } i=n-2\end{cases}
$$

Then it is straightforward to verify that $\phi$ is a good coloring for $(X, F)$.

\section{THE PROOF OF THEOREM 3.2}

In the remainder of the article, $(X, F)$ is a minimum counterexample to Theorem 3.2. By Lemmas 4.1 and 4.2, $|X|=n \geq 2 k+3$. Recall from the sketch of the proof of Theorem 3.2, we need to prove that $|F(i)| \geq q_{i}^{F}$ for all $i \in J_{F}$. First we consider the case that $i \in I_{F}$ (recall that by Lemma 4.4, $I_{F} \neq \varnothing$ ), and prove that in this case we have $|F(i)| \geq q_{i}^{F}+1$.

Suppose $i \in I_{F}$ and $F(i)=[a, a+t]_{2 k+1}$ for some $t \geq 1$. Assume $|F(i)| \leq q_{i}^{F}$. Let $F^{\prime}$ be defined as follows:

$$
F^{\prime}(j)= \begin{cases}F(j), & \text { if } j \neq i-1, i, i+t, \\ F(i-1) \cup[a+k+1, a+t+k]_{2 k+1}, & \text { if } j=i-1, \\ \emptyset, & \text { if } j=i, \\ F(i+t) \cup\{a+t(k+1)\}, & \text { if } j=i+t .\end{cases}
$$

We say $F^{\prime}$ is obtained from $F$ by breaking $F(i)$. Let $F$ be the FCA in Figure 1, then Figure 2 below is the FCA $F^{\prime}$ obtained from $F$ by breaking $F(5)$. The FCA $F^{\prime}$ is obtained from $F$ by removing the vertices in dotted circles and adding the vertices in squares.

Lemma 5.1. Suppose $i \in I_{F},|F(i)| \leq q_{i}^{F}$ and $F^{\prime}$ is obtained from $F$ by breaking $F(i)$. Then $\left(X, F^{\prime}\right)$ is a valid $F C A$ and dominates $(X, F)$. 


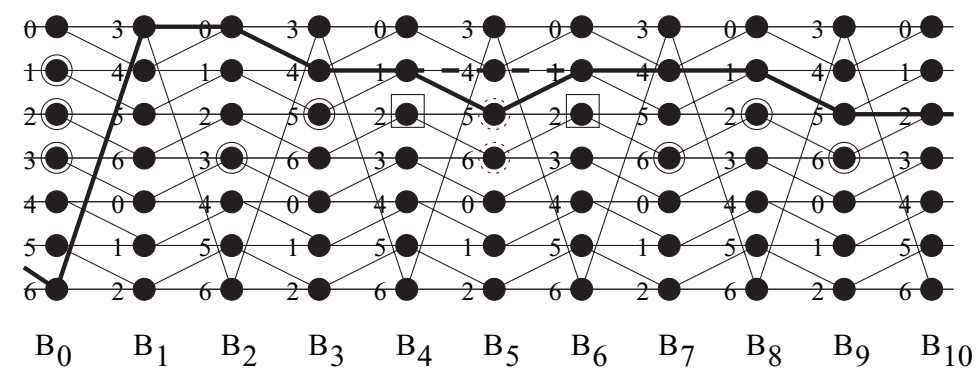

Figure 2. $F^{\prime}$ is obtained from $F$ (in Fig. 1) by breaking $F(5)$.

Proof. It is straightforward to verify that $\left(X, F^{\prime}\right)$ is a valid FCA. We prove that $F^{\prime}$ dominates $F$. Let $\phi^{\prime}$ be a good coloring of $F^{\prime}$. If $\phi^{\prime}\left(x_{i}\right) \notin F(i)$, then $\phi^{\prime}$ is also good for $F$.

Assume $\phi^{\prime}\left(x_{i}\right) \in F(i)$. Since $\phi^{\prime}\left(x_{i-1}\right) \notin F^{\prime}(i-1)$, and $\phi^{\prime}\left(x_{i-1}\right)$ is adjacent to $\phi^{\prime}\left(x_{i}\right)$, it follows from the definition of $F^{\prime}$ that $\phi^{\prime}\left(x_{i}\right)=a$ or $a+t$. Without loss of generality, assume that $\phi^{\prime}\left(x_{i}\right)=a$. Then $\phi^{\prime}\left(x_{i-1}\right)=a+k$ (as $a+k+1 \in F^{\prime}(i-$ 1)). For $j=1,2, \ldots, t$,

$$
\phi^{\prime}\left(x_{i+j}\right) \in\left\{\phi^{\prime}\left(x_{i+j-1}\right)+k, \phi^{\prime}\left(x_{i+j-1}\right)+k+1\right\} .
$$

If for all $j=1,2, \ldots, t, \phi^{\prime}\left(x_{i+j}\right)=\phi^{\prime}\left(x_{i+j-1}\right)+k+1$, then we would have $\phi^{\prime}\left(x_{i+t}\right)=a+t k+t \in F^{\prime}(i+t)$, in contrary to the assumption that $\phi^{\prime}$ is a good coloring for $\left(X, F^{\prime}\right)$. Therefore there is an index $j \in 1,2, \ldots, t$ such that $\phi^{\prime}\left(x_{i+j}\right)=$ $\phi^{\prime}\left(x_{i+j-1}\right)+k$. Let

$$
\phi\left(x_{s}\right)= \begin{cases}\phi^{\prime}\left(x_{s}\right)-1, & \text { if } i \leq s \leq i+j-1 \\ \phi^{\prime}\left(x_{s}\right), & \text { otherwise. }\end{cases}
$$

Then it is easy to verify that $\phi$ is a good coloring for $(X, F)$.

In Figure 2, the thick edges is a good coloring for $F^{\prime}$. The thick broken part indicates the modification of this good coloring to obtain a good coloring of $F$.

Corollary 5.2. For any $i \in I_{F},|F(i)| \geq q_{i}^{F}+1$.

Proof. Assume to the contrary that $i \in I_{F}$ and $|F(i)| \leq q_{i}^{F}$. Let $F_{1}$ be obtained from $F$ by breaking $F(i)$. By Lemma 5.1, $F_{1}$ is a counterexample to Theorem 3.2. It follows from definition that $\left|F_{1}\right| \leq|F|$ and $\left|\sum_{j \in I_{F_{1}}}\right| F_{1}(j)|\leq| \sum_{j \in I_{F}}|F(j)|$. Moreover, $\sum_{j \in I_{F_{1}}}\left|F_{1}(j)\right|=\sum_{j \in I_{F}}|F(j)|$ only if $|F(i-1)|=1$. By the minimality of $(X, F)$, we conclude that $|F(i-1)|=1$. Then $\left|F_{1}(i-1)\right|=|F(i)|$, and easy calculation shows that $q_{i-1}^{F_{1}}=\left|F_{1}(i-1)\right|$. Therefore we can break $F_{1}(i-1)$ to obtain $F_{2}$. The same argument shows that $\left|F_{1}(i-2)\right|=1$. But $F_{1}(i-2)=F(i-$ 2). Repeat this argument, we conclude that $|F(i-j)|=1$ for all $j$ (recall the calculation is modulo $n$ ), which is an obvious contradiction.

It remains to prove that if $|F(j)|=1$, then $q_{j}^{F}=1$, that is, $F(j+1) \neq \emptyset$. For this purpose, we need to consider those $i \in I_{F}$ which are close to $j$. 
Lemma 5.3. Suppose $i \in I_{F}, F(i)=[a, a+t]$ and $F\left(i+q_{i}^{F}\right)=\{b\}$. Then either $b=a+t+1+q_{i}^{F} k$ or $b=a-1+q_{i}^{F}(k+1)$.

Proof. Assume to the contrary that $b \neq a+t+1+q_{i}^{F} k$ and $b \neq a-1+$ $q_{i}^{F}(k+1)$.

Let $X^{\prime}=\left(x_{0}^{\prime}, x_{1}^{\prime}, \ldots, x_{n-3}^{\prime}\right)$ be a cycle of length $n-2$. Let $F^{\prime}$ be defined as

$$
F^{\prime}(j)= \begin{cases}F(j) & \text { if } j \leq i-2 \\ F(j) \cup[a+(k+1), a+t+k]_{2 k+1} & \text { if } j=i-1 \\ \emptyset & \text { if } i \leq j \leq i+q_{i}^{F}-2 \\ F(j+2) & \text { if } i+q_{i}^{F}-1 \leq j \leq n-3 .\end{cases}
$$

It is easy to show that $\left(X^{\prime}, F^{\prime}\right)$ is a valid FCA of $X^{\prime}$.

We shall show that $\left(X^{\prime}, F^{\prime}\right)$ dominates $(X, F)$. Assume $\phi^{\prime}$ is a good coloring for $\left(X^{\prime}, F^{\prime}\right)$. Since $\phi^{\prime}\left(x_{i-1}^{\prime}\right) \notin[a+k+1, a+k+t]_{2 k+1}$, we conclude that $\phi^{\prime}\left(x_{i}^{\prime}\right) \notin$ $[a+1, a+t-1]_{2 k+1}$.

If $\phi^{\prime}\left(x_{i}^{\prime}\right) \notin\{a, a+t\}$, then $\phi^{\prime}\left(x_{i}^{\prime}\right) \notin F(i)$. Let $s \notin F\left(i+q_{i}^{F}\right)$ be a color which is adjacent to $\phi^{\prime}\left(x_{i+q_{i}^{\prime}-1}^{\prime}\right)\left(\right.$ as $\left|F\left(i+q_{i}^{F}\right)\right|=1$, the color $s$ exists). Let

$$
\phi\left(x_{j}\right)= \begin{cases}\phi^{\prime}\left(x_{j}^{\prime}\right) & \text { if } j \leq i+q_{i}^{F}-1 \\ s & \text { if } j=i+q_{i}^{F} \\ \phi^{\prime}\left(x_{j-2}^{\prime}\right) & \text { if } i+q_{i}^{F}+1 \leq j \leq n-1 .\end{cases}
$$

Then it is straightforward to verify that $\phi$ is a good coloring for $F$. Thus we assume $\phi^{\prime}\left(x_{i}^{\prime}\right) \in\{a, a+t\}$. Without loss of generality, assume $\phi^{\prime}\left(x_{i}^{\prime}\right)=a+t$. Since $a+$ $t+k \in F^{\prime}(i-1)$, we have $\phi^{\prime}\left(x_{i-1}^{\prime}\right)=a+t+k+1$.

A similar argument as above shows that $\phi^{\prime}\left(x_{i+q_{i}^{\prime}-2}^{\prime}\right)=b$.

If there is an index $1 \leq j \leq q_{i}^{F}-2$ such that $\phi^{\prime}\left(x_{i+j}\right)=\phi^{\prime}\left(x_{i+j-1}^{\prime}\right)+k+1$, then let $\phi^{\prime \prime}$ be defined as

$$
\phi^{\prime \prime}\left(x_{s}^{\prime}\right)= \begin{cases}\phi^{\prime}\left(x_{s}^{\prime}\right)+1, & \text { if } i \leq s \leq i+j-1 \\ \phi^{\prime}\left(x_{s}^{\prime}\right), & \text { otherwise }\end{cases}
$$

Then $\phi^{\prime \prime}$ is a good coloring for $\left(X^{\prime}, F^{\prime}\right)$ for which $\phi^{\prime \prime}\left(x_{i}^{\prime}\right) \notin F(i)$, which is a case discussed already. Thus we assume that $\phi^{\prime}\left(x_{i+j}^{\prime}\right)=\phi^{\prime}\left(x_{i+j-1}^{\prime}\right)+k$ for $1 \leq j \leq$ $q_{i}^{F}-2$. In particular, $b=\phi^{\prime}\left(x_{i+q_{i}^{\prime}-2}^{\prime}\right)=a+t+\left(q_{i}^{F}-2\right) k=a+t+q_{i}^{F} k-2 k=$ $a+t+1+q_{i}^{F} k$ (recall colors are modulo $2 k+1$ ).

In Figure $3, X^{\prime}$ is obtained from $X$ by removing two columns, namely $B_{5}$ and $B_{6}$, and $F^{\prime}$ is obtained from $F$ by removing the vertices in dotted circle, and adding vertices in squares. The solid thick edges form the coloring cycle corresponding to $\phi^{\prime}$. The broken thick edges are the modified part of $\phi^{\prime}$ to obtain $\phi^{\prime \prime}$. The dotted edges indicate modifications to obtain the coloring cycle for $\phi$.

Suppose $i \in I_{F}$ and $p_{i}^{F}>q_{i}^{F}$. Assume

$$
\left[i, i+p_{i}^{F}\right] \cap J_{F}=\left\{\beta_{0}^{i}, \beta_{1}^{i}, \ldots, \beta_{t_{i}}^{i}\right\},
$$




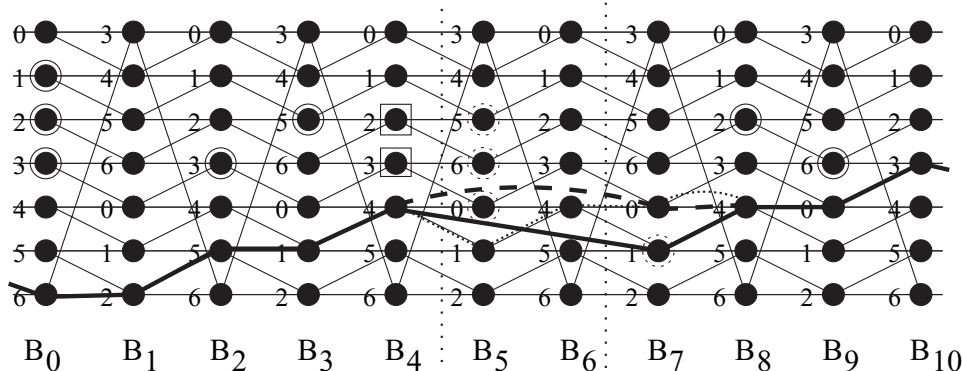

Figure 3. An illustration of the proof of Lemma 5.3.

where $t_{i} \geq 2$ and $i+q_{i}^{F}=\beta_{1}^{i}$. Assume $F(i)=[a, a+t]$ and for $j=1,2, \ldots, t_{i}-$ $1, F\left(\beta_{j}^{i}\right)=\left\{b_{j}\right\}$. By Lemma 5.3, either $b_{1}=a+t+1+q_{i}^{F} k$ or $b_{1}=a-1+$ $q_{i}^{F}(k+1)$. Without loss of generality, assume that $b_{1}=a+t+1+q_{i}^{F} k$.

Lemma 5.4. For $j=1,2, \ldots, t_{i}-1, \quad \beta_{j+1}^{i}=\beta_{j}^{i}+1$. Moreover, for $j=$ $1,2, \ldots, t_{i}-2, b_{j+1}=b_{j}+k+1$.

Proof. Assume the lemma is false. Let $t \geq 1$ be the smallest index such that either

$$
t \leq t_{i}-1 \quad \text { and } \quad \beta_{t+1}^{i} \neq \beta_{t}^{i}+1
$$

or

$$
t \leq t_{i}-2, \beta_{t+1}^{i}=\beta_{t}^{i}+1 \text { but } b_{t+1} \neq b_{t}+k+1
$$

Case 1. $\beta_{t+1}^{i} \neq \beta_{t}^{i}+1$. Then $F\left(\beta_{t}^{i}+1\right)=\emptyset$. Let $F^{\prime}$ be the FCA defined as follows:

$$
F^{\prime}(j)= \begin{cases}F(j) & \text { if } j \neq \beta_{t}^{i}, \beta_{t}^{i}+1 \\ \emptyset & \text { if } j=\beta_{t}^{i} \\ \left\{b_{t}+k\right\} & \text { if } j=\beta_{t}^{i}+1\end{cases}
$$

It is easy to verify that $F^{\prime}$ is a valid FCA. We shall prove that $\left(X, F^{\prime}\right)$ dominates $(X, F)$. Let $\phi^{\prime}$ be a good coloring for $\left(X, F^{\prime}\right)$. If $\phi^{\prime}\left(x_{\beta_{t}^{i}}\right) \neq b_{t}$, then $\phi^{\prime}$ is a good coloring for $F$, we are done.

Assume $\phi^{\prime}\left(x_{\beta_{t}^{i}}\right)=b_{t}$. Since $F^{\prime}\left(\beta_{t}^{i}+1\right)=\left\{b_{t}+k\right\}$, and $\phi^{\prime}\left(x_{\beta_{t}^{i}+1}\right) \notin F^{\prime}\left(\beta_{t}^{i}+1\right)$, it follows that $\phi^{\prime}\left(x_{\beta_{t}^{i}+1}\right)=b_{t}+k+1$.

If $t \geq 2$, then by the minimality of $t$, we have $\beta_{t-1}^{i}=\beta_{t}^{i}-1$ and $b_{t}=b_{t-1}+k+$ 1 , that is, $b_{t-1}=b_{t}+k$. This implies that $\phi^{\prime}\left(x_{\beta_{t}^{i}-1}\right)=b_{t}+k+1$.

If $t=1$, then since $F(i)=[a, a+t]$ and $t \geq q_{i}^{F}$ (by Corollary 5.2) and $b_{1}=a+t+1+q_{i}^{F} k$, straightforward calculation shows that $\phi^{\prime}\left(x_{\beta_{1}^{i}-1}\right)=b_{t}+k$ would imply that $\phi^{\prime}\left(x_{i}\right) \in F(i)$, which is a contradiction. Therefore we also have $\phi^{\prime}\left(x_{\beta_{1}^{i}-1}\right)=b_{t}+k+1$. 
Let

$$
\phi\left(x_{j}\right)= \begin{cases}\phi^{\prime}\left(x_{j}\right) & \text { if } j \neq \beta_{t}^{i} \\ \phi^{\prime}\left(x_{\beta_{t}^{i}}\right)+1=b_{t}+1 & \text { if } j=\beta_{t}^{i}\end{cases}
$$

Then $\phi$ is a good coloring for $(X, F)$. This proves that $\left(X, F^{\prime}\right)$ dominates $(X, F)$. However, $|F|=\left|F^{\prime}\right|, \sum_{i \in I_{F}}|F(i)|=\sum_{i \in I_{F^{\prime}}}\left|F^{\prime}(i)\right|$ and $\sum_{i \in I_{F^{\prime}}} q_{i}=\sum_{i \in I_{F}} q_{i}-1$. This is in contrary to the minimality of $(X, F)$.

Case 2. $t \leq t_{i}-2$ and $\beta_{t+1}^{i}=\beta_{t}^{i}+1$ but $b_{t+1} \neq b_{t}+k+1$.

If $b_{t+1}=b_{t}+k$, then let $F^{\prime}$ be the FCA defined as follows:

$$
F^{\prime}(j)= \begin{cases}F(j) & \text { if } j \neq \beta_{t}^{i}+1 \\ \emptyset & \text { if } j=\beta_{t}^{i}+1\end{cases}
$$

It is routine to verify that $F^{\prime}$ is a valid FCA, Now we show that $\left(X, F^{\prime}\right)$ dominates $(X, F)$. Let $\phi^{\prime}$ be a good coloring for $\left(X, F^{\prime}\right)$. If $\phi^{\prime}\left(x_{\beta_{t}^{i}+1}\right) \neq b_{t+1}$, then $\phi^{\prime}$ is a good coloring for $(X, F)$, we are done. Assume $\phi^{\prime}\left(x_{\beta_{t}^{i}+1}\right)=b_{t+1}=b_{t}+k$. By the minimality of $t$, we know that $b_{j+1}=b_{j}+k+1$ for $j=1,2, \ldots, t-1$. This implies that $\phi^{\prime}\left(x_{\beta_{j}^{i}}\right)=b_{j}-1$ for $j=1,2, \ldots, t-1$. In particular, $\phi^{\prime}\left(x_{\beta_{1}^{i}}\right)=$ $b_{1}-1$. This is a contradiction, because straightforward calculation shows that $\phi^{\prime}\left(x_{\beta_{1}^{i}}\right)=b_{1}-1$ implies that $\phi^{\prime}\left(x_{i}\right) \in F(i)$ (using the fact that $|F(i)| \geq q_{i}^{F}+1$ ). This proves that $\left(X, F^{\prime}\right)$ dominates $(X, F)$. As $\left|F^{\prime}\right|<|F|$, this is in contrary to the minimality of $(X, F)$.

If $b_{t+1} \neq b_{t}+k$, then $b_{t+1}$ is not adjacent to $b_{t}$. Let $X^{\prime}=\left(x_{0}^{\prime}, x_{1}^{\prime}, \ldots, x_{n-3}^{\prime}\right)$ be a cycle of length $n-2$. Define an FCA $F^{\prime}$ of $X^{\prime}$ as

$$
F^{\prime}(j)= \begin{cases}F(j) & \text { if } j \leq \beta_{t}^{i}-1 \\ F(j+2) & \text { if } \beta_{t}^{i}+1 \leq j \leq n-3\end{cases}
$$

Then the same argument as the proof of Lemma 4.3 shows that $\left(X^{\prime}, F^{\prime}\right)$ is a valid FCA which dominates $(X, F)$.

Combining Corollary 5.2 and Lemma 5.4, we have proved that $|F(i)| \geq q_{i}^{F}$ for all $i$, which implies that $n=\sum_{i=0}^{n-1} q_{i}^{F} \leq \sum_{i=0}^{n-1}|F(i)|$, in contrary to our assumption. This completes the proof of Theorem 3.2.

\section{REFERENCES}

[1] O. V. Borodin, S. J. Kim, A. V. Kostochka, and D. B. West, Homomorphisms from sparse graph with large girth, J Combin Theory Ser B 90(1) (2004), $147-159$.

[2] A. Galluccio, L. Goddyn, and P. Hell, High-girth graphs avoiding a minor are nearly bipartite, J Combin Theory Ser B 83 (2001), 1-14.

[3] M. DeVos, Personal Communication at Workshop on Flows and Cycles, Simon Fraser University, 2000. 
[4] G. Fijavž, M. Juvan, B. Mohar, and R. Škrekovski, Circular colorings of planar graphs with prescribed girth, manuscript (2001).

[5] F. Jaeger, On circular flows in graphs, Finite and Infinite Sets (Eger, 1981), Colloquia Mathematica Societatis János Bolyai 37, North Holland (1984), 391-402.

[6] F. Jaeger, "Nowhere-zero flow problems," Selected Topics in Graph Theory 3 (L. W. Beineke and R. J. Wilson, editors), Academic Press, London 1988, pp. 71-95.

[7] W. Klostermeyer and C. Q. Zhang, $(2+\epsilon)$-coloring of planar graphs with large odd girth, J Graph Theory 33 (2000), 109-119.

[8] Z. Pan and X. Zhu, Density of the circular chromatic numbers of series-parallel graphs, J Graph Theory 46 (2004), 57-68.

[9] C. Thomassen, Every planar graph is 5-choosable, J Combin Theory Ser B 62 (1994), 180-181.

[10] C. Thomassen, 3-list-coloring planar graphs of girth at least 5, J Combin Theory Ser B 64 (1995), 101-107.

[11] A. Vince, Star chromatic number, J Graph Theory 12 (1988), 551-559.

[12] X. Zhu, The circular chromatic number of planar graphs of large odd girth, Electronic J Combin (2001), \#R25.

[13] X. Zhu, Circular chromatic number: A survey, Discrete Math 229(1-3) (2001), 371-410.

[14] X. Zhu, "Recent developments in circular coloring of graphs," Topics in Discrete Mathematics (M. Kazar, J.Kratochvil, M. Loeble, J. Matoušek, R. Thomas, and P. Valtr, editors) Springer, Berlin, Heidelberg, New York. 2006, pp. 497-550.

[15] X. Zhu, Circular choosability of graphs, J Graph Theory 48 (2005), 210-218. 